\title{
The Double Chooz experiment
}

\author{
Christian Buck* on behalf of the Double Chooz Collaboration \\ Max-Planck-Institut für Kernphysik Heidelberg, 69117 Heidelberg, Germany \\ E-mail: Christian.Buck@mpi-hd.mpg.de
}

\begin{abstract}
The main goal of the Double Chooz reactor neutrino experiment is a precision measurement of the neutrino mixing angle $\theta_{13}$. This smallest of the three known mixing angles is determined from the disappearance of electron antineutrinos emitted by two nuclear reactors in Chooz, France. Neutrino interactions with the protons in an organic liquid scintillator produce a coincidence signal of a prompt positron and a delayed neutron allowing efficient background suppression. The reactor neutrino flux is measured in two detectors at different distances to the source. The near detector at $400 \mathrm{~m}$ from the reactors provides a reference measurement almost without an oscillation effect. The far detector at $1.05 \mathrm{~km}$ distance is located close to the first oscillation maximum. In this multi-detector configuration the flux uncertainty becomes negligible. A precise measurement of $\theta_{13}$ with the running reactor neutrino experiments is a crucial input for upcoming projects sensitive to $\mathrm{CP}$-violation and atmospheric mass hierarchy observables.
\end{abstract}

Neutrino Oscillation Workshop

4 - 11 September, 2016

Otranto (Lecce, Italy)

${ }^{*}$ Speaker. 


\section{The Double Chooz detectors}

Nuclear reactors are a strong and pure source of electron antineutrinos well suited to study neutrino properties. A new generation of reactor neutrino experiments, Double Chooz (DC) [1], Daya Bay [2] and RENO [3] were able to determine the neutrino mixing $\theta_{13}$. The key feature of these experiments is the concept of having near and far detectors providing a pure measurement of $\theta_{13}$ without relying on flux predictions. The near DC detector at a distance of about $400 \mathrm{~m}$ to the reactors measures the neutrino rate at a position where the oscillation effect is small. The far detector at about $1 \mathrm{~km}$ distance sits close to the first oscillation minimum and detects the disappearance of the electron antineutrinos. At such a rather simple site configuration with just two reactors and two detectors almost at iso-flux positions, the flux related uncertainty becomes negligible compared to other uncertainties as efficiency and backgrounds. Moreover, the experiment profits from the opportunity to measure the backgrounds in periods with both reactors off [4].

The design of the inner DC detectors is identical in near and far position. The neutrinos are detected in an inverse beta decay reaction on protons of the hydrogen atoms in an organic liquid target scintillator (Target). The interaction signature of neutrinos with energies above the reaction threshold of $1.8 \mathrm{MeV}$ is a coincidence signal of a prompt positron and a delayed neutron capture. From the measured positron energy deposition in the Target the neutrino energy can be extracted. To keep the coincidence time short (about $30 \mu \mathrm{s}$ ) and to increase the energy of the gammas emitted in the neutron capture $(8 \mathrm{MeV})$, the Target is doped with $1 \mathrm{~g} / \mathrm{l}$ of gadolinium $(\mathrm{Gd})$. The detector response $(<1 \%$ variation per year) and neutron capture efficiency $(<0.2 \%$ variation per year) were found to be stable for the several years of data taking until now.

The Target liquids with a mass of 8.3 tons each are contained in $8 \mathrm{~mm}$ thick cylindrical acrylic vessels. They are surrounded by about 18 tons of Gd-free liquid scintillator called "Gamma Catcher" (GC), which detects Gammas produced in the neutrino reaction and escaping the Target. The liquids of the Inner Detectors are completed by 80 tons non-scintillating buffer oil outside the acrylic vessel of the GC. This mineral oil is contained in a steel vessel with 390 photomultiplier tubes (PMTs) of 10 inch diameter mounted to the inner walls. Two systems are available for muon detection. The so-called "Inner Veto" (IV) consists of 70 tons of liquid scintillator observed by 78 PMTs of 8 inch. In addition there are flat layers of plastic scintillator strips on top of the detector, the "Outer Veto" (OV).

Several systems are available for detector calibration. There are weekly calibrations with a multi wavelengths LED light injection system to monitor the PMT behaviour. Radioactive gamma sources and a californium (Cf) neutron source were deployed inside the Target along the vertical zaxis in the detector center. The radioactive sources were also positioned at various locations inside the GC routing them along a stainless steel guide tube fixed to the acrylic vessels.

\section{Neutrino selection and backgrounds}

In the Gd analysis of DC [6] we look for the coincidence signal of the prompt positron event and the high energy $(8 \mathrm{MeV})$ delayed event after neutron capture on Gd. To avoid background events due to instrumental light production from the PMT bases, in particular for the far detector [5], it is required that the light signal arrives at the PMTs uniform in space and simultaneous in 
time. Next a muon veto is applied using IV and OV information. The wide prompt and delayed energy windows of $0.5-20 \mathrm{MeV}$ and $4-10 \mathrm{MeV}$ respectively allow for high detection efficiency. A time coincidence $\Delta \mathrm{t}$ cut was set to $0.5-150 \mu \mathrm{s}$. No extra triggers are allowed shortly before and after the prompt candidate. Then the prompt and delayed event must be reconstructed within less than $1 \mathrm{~m}$ distance. Moreover, information obtained by the Inner and Outer Veto systems, from pulse shape and from vertex reconstruction are used to tag and veto background events. With optimized selection cuts the signal to background ratio in DC could be increased to more than 25 .

The small contribution of accidental background from random coincidences in the Gd analysis of DC is measured with high precision by shifting the coincidence time window more than $1 \mathrm{~s}$ from the prompt candidate. However, if neutron captures on hydrogen are included this type of background increases due to the lower delayed energy of $2.2 \mathrm{MeV}$. Therefore, a multivariate analysis based on an artificial neural network (ANN) was implemented for accidental background reduction using the coincidence time, event distance and delayed energy as input parameters [7]. Correlated signals of fast neutrons (FN) or stopping muons (SM) are another source of background. Spallation neutrons produced by muons missing the veto systems of the detector sometimes scatter on protons in the scintillator and mimic a prompt event. If the spallation neutron is captured in the fiducial volume of the detector, it can create the delayed signal as well. The SM events arise from muons stopping and decaying in the detector without being tagged by the veto systems, e.g. because they enter via the chimney. Here the muon track mimics the prompt event and the decay Michel electron the delayed. Background from SM can be largely suppressed using information from the vertex reconstruction, since events in the chimney area differ in PMT time and charge distribution from events inside the Target. The SM background is more critical in the near detector, since there is less overburden and some buffer scintillation at the top part of the detector due to some inter-volume leakage. The spectral shape of the FN background is checked using events, which are tagged by the IV. Moreover, spectral information can be obtained by a high energy fit from the upper end of the neutrino spectrum, a region where correlated background dominates. Finally, there is background from cosmogenically produced $\beta$-n emitters like ${ }^{9} \mathrm{Li}$ or ${ }^{8} \mathrm{He}$. Since the half life of these isotopes is above $100 \mathrm{~ms}$, it is impossible to veto them without introducing significant dead-time. A dedicated veto allows to partially reject $\mathrm{Li}$ events. The spectral shape was determined from $\mathrm{Li}$ candidate events and it was found to be consistent with the one obtained from simulation studies. The $\mathrm{Li}$ background rate is best determined in the rate + shape oscillation fit.

One of the unique features in the DC experiment is the possibility of measuring the background in reactor off periods. So far there were two of these reactor off periods during the period with far detector only data. The reactor off-off result can be used to constrain the total background rate in the neutrino oscillation analyses.

\section{Oscillation results}

In DC the neutrino mixing angle $\theta_{13}$ was determined in several ways. The first analyses were based on a fit including rate and shape information using only events with neutron captures on gadolinium $[1,8,6]$. Later a rate only analysis fitting the observed antineutrino rates for different reactor power conditions was developed. The result of this approach is independent of any background model [9] and yields a simultaneous measurement of the two fit parameters $\theta_{13}$ and total 
background rate. A statistically independent and larger sample of events was obtained by searching for neutrino candidates with the neutrons captured on hydrogen [10, 7]. In this way the target volume could be extended to the GC liquid. All methods provided consistent results.

For the first years of DC the mixing angle $\theta_{13}$ was determined with data from the far detector only. Since beginning of 2015 DC is running with both detectors simultaneously. After nine months of data taking with two detectors we obtained $\sin ^{2} 2 \theta_{13}=0.111 \pm 0.018$, slightly higher than the best fit values of Daya Bay and RENO (about $1.6 \sigma$ difference). The sensitivity of this data sample was limited by the low statistics. The statistical power of the experiment is improved by almost a factor of three by including neutron captures on hydrogen in the neutrino selection. In this way the neutrino rate in the near (far) detector is increasing to about 900 (140) events/day. It was demonstrated that the background level could be kept under control and the signal to background ratio in both detectors is still well above 10 with the new selection. Whereas the DC analyses in the past were dominated by the uncertainty of the reactor flux (one detector only), the upcoming results with the higher statistics including $\mathrm{Gd}+\mathrm{H}$ events are expected to be limited by uncertainties in the detection systematics, in particular the proton number of the GC.

\section{Spectral distortion}

There are some structures in the prompt neutrino spectrum not expected from shape calculations. For example it could be shown in DC that an excess of events as compared to predictions around $5 \mathrm{MeV}$ is correlated with the reactor flux which disfavours explanations based on unknown background contributions [6]. The feature is observed in the $\mathrm{Gd}$ and $\mathrm{H}$ data samples independently and in both DC detectors. The spectral distortion in the 4-6 MeV area is also detected in other experiments as Daya Bay [11] or RENO [12]. The new DC data with the near detector will allow more detailed studies of the neutrino spectrum and might help to find the explanation of this anomalous shape behaviour.

\section{References}

[1] Y. Abe et al. (Double Chooz Collaboration), Phys.Rev.Lett. 108, 131801 (2012).

[2] F.P. An et al. (Daya Bay Collaboration), Phys.Rev.Lett. 108, 171803 (2012).

[3] J.K. Ahn et al. (RENO Collaboration), Phys.Rev.Lett. 108, 191802 (2012).

[4] Y. Abe et al. (Double Chooz Collaboration), Phys.Rev. D 87, 011102(R) (2013).

[5] Y. Abe et al. (Double Chooz Collaboration), JINST 11 (2016) P08001.

[6] Y. Abe et al. (Double Chooz Collaboration), JHEP 10 (2014) 086.

[7] Y. Abe et al. (Double Chooz Collaboration), JHEP 01 (2016) 163.

[8] Y. Abe et al. (Double Chooz Collaboration), Phys.Rev.Lett. D 86 (2012) 052008.

[9] Y. Abe et al. (Double Chooz Collaboration), Phys.Lett. B 735 (2014) 51-56.

[10] Y. Abe et al. (Double Chooz Collaboration), Phys.Lett. B 723 (2013) 66-70.

[11] F.P. An et al. (Daya Bay Collaboration), Phys.Rev.Lett. 116, 061801 (2016).

[12] J.H. Choi et al. (RENO Collaboration), Phys.Rev.Lett. 108, 211801 (2016). 